\title{
Effective potential for composite operators and for auxiliary scalar field in a Nambu-Jona-Lasinio model
}

\author{
Bang-Rong Zhou \\ CCAST (World Laboratory) P.O. Box 8730, Beijing 100080, China and \\ Department of physics, the Graduate School of Chinese Academy of Sciences, Beijing 100039, China*
}

\begin{abstract}
We derive the effective potentials for composite operators in a Nambu-Jona-Lasinio (NJL) model at zero and finite temperature and show that in each case they are equivalent to the corresponding effective potentials based on an auxiliary scalar field. The both effective potentials could lead to the same possible spontaneous breaking and restoration of symmetries including chiral symmetry if the momentum cutoff in the loop integrals is large enough, and can be transformed to each other when the Schwinger-Dyson (SD) equation of the dynamical fermion mass from the fermion-antifermion vacuum (or thermal) condensates is used. The results also generally indicate that two effective potentials with the same single order parameter but rather different mathematical expressions can still be considered physically equivalent if the SD equation corresponding to the extreme value conditions of the two potentials have the same form.
\end{abstract}

\section{INTRODUCTION}

Effective potential [1, 2, 3, 4, 5] is a basic means to research the vacuum of quantum field theory and spontaneous symmetry breaking. It can be derived by means of different approaches. Consider a simple Nambu-Jona-Lasinio (NJL) model [2] of four-fermion interactions

$$
\mathcal{L}(x)=\sum_{k=1}^{N} \bar{\psi}^{k}(x) i \gamma^{\mu} \partial_{\mu} \psi_{k}(x)+\frac{g}{2} \sum_{k=1}^{N}\left[\bar{\psi}^{k}(x) \psi_{k}(x)\right]^{2},
$$

where $\psi_{k}(x)$ are the fermion fields with $N$ "color" components and $g$ is the four-fermion coupling constant. The conventional approach to derive effective potential of the model is to introduce an auxiliary scalar field $\sigma(x)$ [], since the Lagrangian (1) is equivalent to

$$
\mathcal{L}_{\sigma}(x)=\sum_{k=1}^{N} \bar{\psi}^{k}(x) i \gamma^{\mu} \partial_{\mu} \psi_{k}(x)-\sigma(x) \sum_{k=1}^{N} \bar{\psi}^{k}(x) \psi_{k}(x)-\frac{1}{2 g} \sigma^{2}(x),
$$

Then, In terms of a local external source $J(x)$ and the standard procedure, one will obtain from Eq.(2) the effective action and corresponding effective potential. The latter, in the leading order of $1 / N$ expansion, can be expressed by [7, [8]

$$
V_{\sigma}\left(m_{0}\right)=\frac{m_{0}^{2}}{2 g}+2 N \int \frac{i d^{4} l}{(2 \pi)^{4}} \ln \left(1-\frac{m_{0}^{2}}{l^{2}+i \varepsilon}\right),
$$

where the constant "classical field " $\sigma_{c}$ has been identified with the dynamical fermion mass $m_{0}$.

Alternatively, we may have another approach to derive effective potential of the model (1), i.e. the effective action approach for composite operators presented by Conwall, Jackiw and Tomboulis (CJT) 9]. In this approach, instead of introducing an auxiliary scalar field, one considers the fermion propagator as the order parameter of the effective potential. Since the fermion propagator $G(x, y)$ is a bi-local function, one must put in a bi-local external source $K(x, y)$. The effective action is the energy of the system when $G(x, y)$ is fixed. Hence in the derivation of the effective action, the external source $K(x, y)$ must be so selected as to keep $G(x, y)$ to be fixed. In the final result, $G(x, y)$ will be the exact fermion propagator, i.e. no higher order corrections to it. The CJT effective action $\Gamma[G]$ for the models without a basic scalar field can be expressed by $[9,10]$

$$
\Gamma[G]=-i \operatorname{Tr} \ln \left(S G^{-1}\right)-i \operatorname{Tr}\left(S^{-1} G\right)+i \operatorname{Tr} 1+\Gamma_{2}[G],
$$

where

$$
i S^{-1}=i \gamma^{\mu} \partial_{\mu}
$$

* Mailing address. 
i.e. $S$ corresponds to the propagator for free massless fermion. The $\mathrm{Tr}$ is in functional sense. The first three terms in Eq.(4) are the contributions of one-loop diagrams and $\Gamma_{2}[G]$ represents the contributions of two- and more- loop vacuum graphs without fermion self-energy correction, since $G$ is the exact fermion propagator. In a theory of translational invariance, $G(x, y)=G(x-y)$, we can define the effective potential $V[G]$ by

$$
\Gamma[G]=-\Omega V[G],
$$

where $\Omega$ is the space-time volume. In the four-fermion interaction model given by Eq.(1), we will have $V[G]=V\left(m_{0}\right)$, i.e. the order parameter may be replaced by the dynamical fermion mass $m_{0}$.

Several natural and interesting questions follow: whether is the effective potential so derived equivalent to the one from the auxiliary scalar field or not? Whether is there any relation between the two effective potentials? What will it mean if the answers are positive? In this paper we will reply the above questions through calculating CTJ potential of the model (1) and comparing it with the result from the auxiliary scalar field approach. Besides zero temperature case, we will also discuss finite temperature case. The discussions will be conducted in the real-time thermal field theory [11, 12, 13], and this could give us some more insight of that how to calculate CJT effective potential in the real-time formalism of thermal field theory, noting that conventional calculations were made in the imaginary-time formalism [14].

The paper is arranged as follows. In SecII we will derive the CJT potential of the model (1) when temperature $T=0$ and fermionic chemical potential $\mu=0$, discuss spontaneous symmetry breaking and explore the relation between the result and Eq.(3) from the auxiliary scalar field approach. In Sec generalized to the case of finite $T$ and finite $\mu$ and in Sec IV we give our conclusions.

\section{CJT POTENTIAL AT $T=\mu=0$}

In the momentum space, we have

$$
S(p)=i / \not p, G(p)=i /\left(\not p-m_{0}\right), \not p \equiv \gamma^{\mu} p_{\mu},
$$

where $G(p)$ is the exact fermion propagator when the four-fermion interactions exist and the dynamical fermion mass $m_{0}$ should be a constant. When keeping only the vacuum-vacuum diagram up to two-loop order with one four-fermion coupling vertex in $\Gamma_{2}[G]$, we will obtain from Eq.(4) the CJT effective action of the model (1) at $T=\mu=0$

$$
\begin{aligned}
\Gamma[G]= & -i N\left\langle\left[\operatorname{tr} \ln S(p) G^{-1}(p)+\operatorname{tr} S^{-1}(p) G(p)-\operatorname{tr} 1\right]\langle p \mid p\rangle\right\rangle \\
& +\frac{g}{2}\langle\operatorname{tr} G(p)\rangle^{2}(2 \pi)^{4} \delta^{4}(0),
\end{aligned}
$$

where $\operatorname{tr}$ only represents the trace of a spinor matrix, the denotation $\langle\cdots\rangle$ has been used for $\int d^{4} p /(2 \pi)^{4}$. By means of Eq.(6) and the relations $\langle p \mid p\rangle=(2 \pi)^{4} \delta^{4}(0)=\Omega$, we can write Eq.(7) by

$$
\Gamma[G]=-\Omega V\left(m_{0}\right)
$$

where, after the Wick rotation of the integral variable $p$, the effective potential $V\left(m_{0}\right)$ may be expressed by

$$
V\left(m_{0}\right)=-2 N\left\langle\ln \frac{\bar{p}^{2}+m_{0}^{2}}{\bar{p}^{2}}\right\rangle+4 N\left\langle\frac{m_{0}^{2}}{\bar{p}^{2}+m_{0}^{2}}\right\rangle-8 N^{2} g\left\langle\frac{m_{0}}{\bar{p}^{2}+m_{0}^{2}}\right\rangle^{2}
$$

where and afterwards, the denotation $\bar{p}$ will be understood as Euclidean 4 momentum. By the effective potential $V\left(m_{0}\right)$, we may discuss spontaneous symmetry breaking of the model (1). A non-zero order parameter $m_{0}$ in the vacuum state will mean spontaneous breaking of the discrete chiral symmetry $\chi_{D}: \psi_{k}(x) \stackrel{\chi_{D}}{\longrightarrow} \gamma_{5} \psi_{k}(x)$ and the special parities $\mathcal{P}_{j}: \psi_{k}\left(t, \cdots, x^{j}, \cdots\right) \stackrel{\mathcal{P}_{j}}{\longrightarrow} \gamma^{j} \psi_{k}\left(t, \cdots,-x^{j}, \cdots\right)(j=1,2,3)$. It is obtained from Eq.(9) that

$$
\frac{\partial V\left(m_{0}\right)}{\partial m_{0}}=4 N m_{0}\left\langle\frac{\bar{p}^{2}-m_{0}^{2}}{\left(\bar{p}^{2}+m_{0}^{2}\right)^{2}}\right\rangle\left(1-4 N g\left\langle\frac{1}{\bar{p}^{2}+m_{0}^{2}}\right\rangle\right)
$$

with

$$
\left\langle\frac{\bar{p}^{2}-m_{0}^{2}}{\left(\bar{p}^{2}+m_{0}^{2}\right)^{2}}\right\rangle=\frac{m_{0}^{2}}{16 \pi^{2}}\left[\frac{\Lambda^{2}}{m_{0}^{2}}-3 \ln \left(\frac{\Lambda^{2}}{m_{0}^{2}}+1\right)+2 \frac{\Lambda^{2}}{\Lambda^{2}+m_{0}^{2}}\right]
$$


where $\Lambda$ is the 4 dimensional Euclidean momentum cutoff. It is easy to check that

$$
\left\langle\frac{\bar{p}^{2}-m_{0}^{2}}{\left(\bar{p}^{2}+m_{0}^{2}\right)^{2}}\right\rangle>0, \text { when } \frac{\Lambda^{2}}{m_{0}^{2}}>1.82 .
$$

Assuming that $\Lambda$ is large so that Eq.(11) is true, then the extreme value condition $\partial V\left(m_{0}\right) / \partial m_{0}=0$ will be satisfied if 1) $m_{0}=0$ and 2) $m_{0}=m_{01}$, where $m_{01}$ is determined by the gap equation

$$
1-4 N g\left\langle\frac{1}{\bar{p}^{2}+m_{01}^{2}}\right\rangle=0
$$

Then we may verify that

$$
\left.\frac{\partial^{2} V\left(m_{0}\right)}{\partial m_{0}^{2}}\right|_{m_{0}=0}=4 N\left\langle\frac{1}{\bar{p}^{2}}\right\rangle\left(1-4 N g\left\langle\frac{1}{\bar{p}^{2}}\right\rangle\right)
$$

and

$$
\left.\frac{\partial^{2} V\left(m_{0}\right)}{\partial m_{0}^{2}}\right|_{m_{0}=m_{01}}=32 N^{2} g\left\langle\frac{\bar{p}^{2}-m_{01}^{2}}{\left(\bar{p}^{2}+m_{01}^{2}\right)^{2}}\right\rangle\left\langle\frac{m_{01}^{2}}{\left(\bar{p}^{2}+m_{01}^{2}\right)^{2}}\right\rangle>0, \text { if } \frac{\Lambda^{2}}{m_{01}^{2}}>1.82
$$

Obviously, when $1-4 N g\left\langle 1 / \bar{p}^{2}\right\rangle=1-N g \Lambda^{2} / 4 \pi^{2}>0$ or $N g \Lambda^{2} / 4 \pi^{2}<1$, i.e. the four-fermion coupling $g$ is weak, $V\left(m_{0}\right)$ has the only minimum point $m_{0}=0$ thus no spontaneous symmetry breaking occurs in this case; Conversely, when

$$
\frac{N g \Lambda^{2}}{4 \pi^{2}}>1
$$

i.e. the four-fermion coupling $g$ is strong enough, $V\left(m_{0}\right)$ will have a maximum point $m_{0}=0$ and a minimum point $m_{0}=m_{01}$ which is now the non-zero solution of Eq.(12). In this case spontaneous symmetry breaking will occur. Noting that Eq.(15) is compatible with the condition $\Lambda^{2} / m_{01}^{2}>1.82$.

We indicate that the same conclusion can be obtained by the effective potential (3) derived from the auxiliary scalar field approach. In fact, after the Wick rotation, Eq.(3) becomes

$$
V_{\sigma}\left(m_{0}\right)=\frac{m_{0}^{2}}{2 g}-2 N\left\langle\ln \frac{\bar{p}^{2}+m_{0}^{2}}{\bar{p}^{2}}\right\rangle
$$

and it further leads to

$$
\frac{\partial V_{\sigma}\left(m_{0}\right)}{\partial m_{0}}=\frac{m_{0}}{g}\left(1-4 N g\left\langle\frac{1}{\bar{p}^{2}+m_{0}^{2}}\right\rangle\right) .
$$

It is seen by a comparison that Eq.(17) and Eq.(10) are identical except a factor $4 N g\left\langle\left(\bar{p}^{2}-m_{0}^{2}\right) /\left(\bar{p}^{2}+m_{0}^{2}\right)^{2}\right\rangle$. Consequently, as far as symmetry breaking is concerned, $V_{\sigma}\left(m_{0}\right)$ will lead to the same conclusion as $V\left(m_{0}\right)$ when $\Lambda^{2} / m_{0}^{2}>1.82$. We notice that in the auxiliary scalar field approach, the dynamical fermion mass $m_{0}$ comes from the vacuum expectation value of the scalar field $\sigma(x)$ and in the CJT composite operator approach, $m_{0}$ originates from the fermion-antifermion condensates $\langle\bar{\psi} \psi\rangle$ through the relation

$$
m_{0}=-\frac{g}{2}\langle\bar{\psi} \psi\rangle=4 N g\left\langle\frac{m_{0}}{\bar{p}^{2}+m_{0}^{2}}\right\rangle .
$$

Hence if we substitute Eq.(18) into the CJT potential $V\left(m_{0}\right)$ in Eq. $(9)$ and physically this amounts to view $-\frac{g}{2}\langle\bar{\psi} \psi\rangle$ effectively as the vacuum expectation value of an auxiliary scalar field, then we should be able to obtain $V_{\sigma}\left(m_{0}\right)$ from $V\left(m_{0}\right)$. This is in fact true. Comparing Eq.(9) with Eq.(16) we may see that the last two terms in Eq.(9) should correspond to the first term in the right-handed side of Eq.(16). This can be directly verified by putting Eq.(18) into Eq.(9).

We emphasize that the key sectors of the extreme value equations $\partial V\left(m_{0}\right) / \partial m_{0}=0$ and $\partial V_{\sigma}\left(m_{0}\right) / \partial m_{0}=0$ are the same and it is just Eq.(18). This fact indicates that $V\left(m_{0}\right)$ and $V_{\sigma}\left(m_{0}\right)$ are essentially determined by the form of the SD equation (18) and this explains that why they are completely equivalent despite their different expressions. 


\section{CJT POTENTIAL AT FINITE $T$ AND $\mu$}

The extension of the CJT effective potential (4) to finite $T$ and $\mu$ can be expressed in the real-time formalism of thermal field theory by

$$
\begin{aligned}
\Gamma_{T}[G] & =-i \operatorname{Tr}\left[\ln \left(S_{T} G_{T}^{-1}\right)\right]^{11}-i \operatorname{Tr}\left(S_{T}^{-1} G_{T}\right)^{11}+i \operatorname{Tr} 1+\Gamma_{2 T}[G] \\
& =-i N\left\langle\left[\operatorname{tr}\left[\ln S_{T}(p) G_{T}^{-1}(p)\right]^{11}\right\rangle \Omega-i N\left\langle\operatorname{tr}\left[S_{T}^{-1}(p) G_{T}(p)\right]^{11}\right\rangle \Omega+i N\langle\operatorname{tr} 1\rangle \Omega+\Gamma_{2 T}[G],\right.
\end{aligned}
$$

where $S_{T}$ and $G_{T}$ are $2 \times 2$ thermal matrix propagators, the superscript "11" represents the 11 component of the corresponding matrix. In the momentum space, we have 11]

$$
S_{T}(p)=M_{p} \tilde{S}(p) M_{p}, G_{T}(p)=M_{p} \tilde{G}(p) M_{p}
$$

with the thermal transformation matrix $M_{p}$ defined by

$$
M_{p}=\left(\begin{array}{cc}
\cos \theta_{p} & -e^{\beta \mu / 2} \sin \theta_{p} \\
e^{-\beta \mu / 2} \sin \theta_{p} & \cos \theta_{p}
\end{array}\right), \sin ^{2} \theta_{p}=\frac{\theta\left(p^{0}\right)}{e^{\beta\left(p^{0}-\mu\right)}+1}+\frac{\theta\left(-p^{0}\right)}{e^{\beta\left(-p^{0}+\mu\right)}+1}, \beta=1 / T
$$

and

$$
\tilde{S}(p)=\left(\begin{array}{cc}
S(p) & 0 \\
0 & S^{*}(p)
\end{array}\right), \tilde{G}(p)=\left(\begin{array}{cc}
G(p) & 0 \\
0 & G^{*}(p)
\end{array}\right)
$$

where

$$
\begin{aligned}
& S(p)=i /(\not p+i \varepsilon), S^{*}(p)=-i /(\not p-i \varepsilon) \\
& G(p)=i /(\not p-m+i \varepsilon), G^{*}(p)=-i /(\not p-m-i \varepsilon)
\end{aligned}
$$

and $m \equiv m(T, \mu)$ is the dynamical fermion mass at finite $T$ and $\mu$. Noting that, by Eq.(20),

$$
\ln S_{T}(p) G_{T}^{-1}(p)=\ln M_{p} \tilde{S}(p) \tilde{G}^{-1}(p) M_{p}^{-1}=M_{p} \ln \tilde{S}(p) \tilde{G}^{-1}(p) M_{p}^{-1} .
$$

The last step can be checked by a formal power series expansion of the ln expression. We may obtain from Eqs. (21)-(24) that

$$
\begin{aligned}
\operatorname{tr}\left[\ln S_{T}(p) G_{T}^{-1}(p)\right]^{11} & =\cos ^{2} \theta_{p} \operatorname{tr} \ln \left[S(p) G^{-1}(p)\right]+\sin ^{2} \theta_{p} \operatorname{tr} \ln \left[S^{*}(p) G^{-1 *}(p)\right] \\
& =2 \cos ^{2} \theta_{p} \ln \left(1-\frac{m^{2}}{p^{2}+i \varepsilon}\right)+2 \sin ^{2} \theta_{p} \ln \left(1-\frac{m^{2}}{p^{2}-i \varepsilon}\right)
\end{aligned}
$$

Similarly, we may obtain

$$
\begin{aligned}
\operatorname{tr}\left[S_{T}^{-1}(p) G_{T}(p)\right]^{11} & =\operatorname{tr}\left[M_{p}^{-1} \tilde{S}^{-1}(p) \tilde{G}(p) M_{p}\right]^{11} \\
& =\cos ^{2} \theta_{p} \operatorname{tr}\left[S^{-1}(p) G(p)\right]+\sin ^{2} \theta_{p} \operatorname{tr}\left[S^{-1 *}(p) G^{*}(p)\right] \\
& =4\left[\cos ^{2} \theta_{p} \frac{p^{2}}{p^{2}-m^{2}+i \varepsilon}+\sin ^{2} \theta_{p} \frac{p^{2}}{p^{2}-m^{2}-i \varepsilon}\right]
\end{aligned}
$$

To two-loop order of the four-fermion interactions, we can calculate

$$
\begin{aligned}
\Gamma_{2 T}[G] & =\frac{g}{2}\left(\operatorname{Tr} G_{T}^{11}\right)^{2}(2 \pi)^{4} \delta^{4}(0) \\
& =\frac{g}{2} N^{2}\left\langle\operatorname{tr} G_{T}^{11}(p)\right\rangle^{2} \Omega \\
& =\frac{g}{2} N^{2}\left\langle\operatorname{tr}\left[\cos ^{2} \theta_{p} G(p)-\sin ^{2} \theta_{p} G^{*}(p)\right]\right\rangle^{2} \Omega \\
& =-8 g N^{2}\left\langle\cos ^{2} \theta_{p} \frac{m}{p^{2}-m^{2}+i \varepsilon}+\sin ^{2} \theta_{p} \frac{m}{p^{2}-m^{2}-i \varepsilon}\right\rangle^{2} \Omega .
\end{aligned}
$$

Substituting Eqs.(25)-(27) into Eq.(19), we will get the effective action at finite $T$ and $\mu$

$$
\Gamma_{T}[G]=-\Omega V(T, \mu, m)
$$


where

$$
\begin{aligned}
V(T, \mu, m)= & i 2 N\left\langle\cos ^{2} \theta_{p} \ln \left(1-\frac{m^{2}}{p^{2}+i \varepsilon}\right)+\sin ^{2} \theta_{p} \ln \left(1-\frac{m^{2}}{p^{2}-i \varepsilon}\right)\right\rangle \\
& +i 4 N\left\langle\cos ^{2} \theta_{p} \frac{p^{2}}{p^{2}-m^{2}+i \varepsilon}+\sin ^{2} \theta_{p} \frac{p^{2}}{p^{2}-m^{2}-i \varepsilon}\right\rangle \\
& +8 g N^{2}\left\langle\cos ^{2} \theta_{p} \frac{m}{p^{2}-m^{2}+i \varepsilon}+\sin ^{2} \theta_{p} \frac{m}{p^{2}-m^{2}-i \varepsilon}\right\rangle^{2}
\end{aligned}
$$

is the effective potential at finite $T$ and $\mu$ with the order parameter $m$. By using Eq.(29), we can discuss spontaneous symmetry breaking of the model at finite $T$ and $\mu$. It is found out that

$$
\frac{\partial V(T, \mu, m)}{\partial m}=4 N\left\langle\frac{i\left(p^{2}+m^{2}\right)}{\left(p^{2}-m^{2}+i \varepsilon\right)^{2}}\right\rangle m\left(1-4 N g\left\langle\frac{i}{l^{2}-m^{2}+i \varepsilon}-2 \pi \delta\left(l^{2}-m^{2}\right) \sin ^{2} \theta_{l}\right\rangle\right) .
$$

In view of Eq.(11), we will have $\left\langle i\left(p^{2}+m^{2}\right) /\left(p^{2}-m^{2}+i \varepsilon\right)^{2}\right\rangle>0$, if $\Lambda^{2} / m^{2}>1.82$ (after Wick rotation) is assumed. Then the extreme value condition $\partial V(T, \mu, m) / \partial m=0$ will correspond to the equation

$$
m\left(1-4 N g\left\langle\frac{i}{l^{2}-m^{2}+i \varepsilon}-2 \pi \delta\left(l^{2}-m^{2}\right) \sin ^{2} \theta_{l}\right\rangle\right)=0
$$

which is just the Schwinger-Dyson equation $m=-(g / 2)\langle\bar{\psi} \psi\rangle_{T}$ obeyed by the dynamical fermion mass $m$ at finite $T$ and $\mu$, where $\langle\bar{\psi} \psi\rangle_{T}$ is the thermal condensates at temperature $T$ [12]. The possible solutions of Eq.(31) are that 1) $m=0$ and 2) $m=m_{1}$, where $m_{1}$ obeys the gap equation

$$
1-4 N g\left\langle\frac{i}{l^{2}-m_{1}^{2}+i \varepsilon}-2 \pi \delta\left(l^{2}-m_{1}^{2}\right) \sin ^{2} \theta_{l}\right\rangle=0 .
$$

We may further find out that

$$
\left.\frac{\partial^{2} V(T, \mu, m)}{\partial m^{2}}\right|_{m=0}=\frac{N \Lambda^{2}}{4 \pi^{2}}\left[1-\frac{N g \Lambda^{2}}{4 \pi^{2}}+\frac{N g}{2 \pi^{2}} F_{3}(T, \mu, m=0)\right]
$$

with the denotations

$$
F_{3}(T, \mu, m)=2 T^{2} \int_{0}^{\infty} \frac{d x x^{2}}{\sqrt{x^{2}+y^{2}}}\left[\frac{1}{\exp \left(\sqrt{x^{2}+y^{2}}-r\right)+1}+(-r \rightarrow r)\right], y=\frac{m}{T}, r=\frac{\mu}{T}
$$

and

$$
\left.\frac{\partial^{2} V(T, \mu, m)}{\partial m^{2}}\right|_{m=m_{1}}=32 N^{2} g\left\langle\frac{\bar{p}^{2}-m_{1}^{2}}{\left(\bar{p}^{2}+m_{1}^{2}\right)^{2}}\right\rangle\left\langle\frac{m_{1}^{2}}{\left(\bar{p}^{2}+m_{1}^{2}\right)^{2}}\right\rangle>0, \text { if } \frac{\Lambda^{2}}{m_{1}^{2}}>1.82 .
$$

The gap equation (32) can be changed into

$$
1-\frac{N g \Lambda^{2}}{4 \pi^{2}}+\frac{N g}{2 \pi^{2}}\left[F_{3}\left(T, \mu, m_{1}\right)+\frac{m_{1}^{2}}{2} \ln \frac{\Lambda^{2}+m_{1}^{2}}{m_{1}^{2}}\right]=0
$$

and it is noted that $F_{3}(T, \mu, m)$ increases as $m$ goes down. We may see from Eq.(33) that if

$$
1-\frac{N g \Lambda^{2}}{4 \pi^{2}}+\frac{N g}{2 \pi^{2}} F_{3}(T, \mu, m=0)<0,
$$

then $m=0$ will be a maximum point of $V(T, \mu, m)$ and it is easy to verify that in this case Eq.(35) could have a solution $m_{1} \neq 0$ which , by Eq.(34), is a minimum point of $V(T, \mu, m)$, hence we will have spontaneous symmetry breaking at finite $T$ and $\mu$. However, as $T$ and/or $\mu$ further increase, $F_{3}(T, \mu, m)$ will go up, and by Eq.(35), $m_{1}$ will go down and finally to zero. As a result, we will obtain the equation satisfied by critical temperature $T_{c}$ and the critical chemical potential $\mu_{c}$

$$
1-\frac{N g \Lambda^{2}}{4 \pi^{2}}+\frac{N g}{2 \pi^{2}} F_{3}\left(T_{c}, \mu_{c}, m_{1}=0\right)=0
$$


At $T_{c}$ and $\mu_{c}$, we get from Eqs. (33) and (36)

$$
\left.\frac{\partial^{2} V\left(T_{c}, \mu_{c}, m\right)}{\partial m^{2}}\right|_{m=0}=0
$$

If $T$ and/or $\mu$ continue to go up and $F_{3}(T, \mu, m=0)$ will also further increase, then we will be led to

$$
\left.\frac{\partial^{2} V\left(T_{c}, \mu_{c}, m\right)}{\partial m^{2}}\right|_{m=0}>0
$$

This indicates that at the critical point $T_{c}$ and $\mu_{c}, m=0$ will change from being a maximum into a minimum, the order parameter $m$ also varies from nonzero to zero. $V(T, \mu, m)$ will be left the only minimum point $m=0$ and this means that the discrete chiral symmetry $\chi_{D}$ and the special parities $\mathcal{P}_{j}(j=1,2,3)$ which are spontaneously broken at $T=\mu=0$ and $T<T_{c}$ and/or $\mu<\mu_{c}$ will be restored. However, the above discussions do not involve the order of the phase transition. For the latter more demonstrations are needed [15].

Similar to the zero temperature case, the above conclusions coming from the CJT potential $V(T, \mu . m)$ can also be reached by the effective potential $V_{\sigma}(T, \mu, m)$ based on the auxiliary scalar field. The effective potential of a NJL model at finite $T$ and $\mu$ based on auxiliary field was derived for the first time in Ref. 16]. For the model (1), $V_{\sigma}(T, \mu, m)$ and relevant expressions can be written by

$$
\begin{gathered}
V_{\sigma}(T, \mu, m)=\frac{m^{2}}{2 g}+i 2 N\left\langle\cos ^{2} \theta_{p} \ln \left(1-\frac{m^{2}}{p^{2}+i \varepsilon}\right)+\sin ^{2} \theta_{p} \ln \left(1-\frac{m^{2}}{p^{2}-i \varepsilon}\right)\right\rangle \\
\frac{\partial V_{\sigma}(T, \mu, m)}{\partial m}=\frac{m}{g}\left(1-4 N g\left\langle\frac{i}{p^{2}-m^{2}+i \varepsilon}-2 \pi \delta\left(p^{2}-m^{2}\right) \sin ^{2} \theta_{p}\right\rangle\right) \\
\left.\frac{\partial^{2} V_{\sigma}(T, \mu, m)}{\partial m^{2}}\right|_{m=0}=\frac{1}{g}\left[1-\frac{N g \Lambda^{2}}{4 \pi^{2}}+\frac{N g}{2 \pi^{2}} F_{3}(T, \mu, m=0)\right] \\
\left.\frac{\partial^{2} V_{\sigma}(T, \mu, m)}{\partial m^{2}}\right|_{m=m_{1}}=8 m_{1}^{2}\left\langle\frac{-i}{\left(p^{2}-m_{1}^{2}+i \varepsilon\right)^{2}}\right\rangle>0,
\end{gathered}
$$

where $m_{1}$ is the non-zero solution of $\partial V_{\sigma}(T, \mu, m) / \partial m=0$. Obviously Eqs.(37)-(40) will reproduce the total conclusions from the CJT potential $V(T, \mu, m)$. This indicates that at finite $T$ and $\mu$, the two potentials are also completely equivalent. In fact, if substituting the SD equation (31) from the thermal condensates $\langle\bar{\psi} \psi\rangle_{T}$ into Eq.(29), then we will reduce $V(T, \mu, m)$ to $V_{\sigma}(T, \mu, m)$ given by Eq.(37). This shows that the following two approaches will lead to physically identical results: one is that after the thermal condensates $\langle\bar{\psi} \psi\rangle_{T}$ are formed, to consider them as an effective constant scalar field to generate the dynamical fermion mass $m$, and another one is that from the beginning of the discussions, to replace the four-fermion interactions by the effective Yukawa coupling between an auxiliary scalar field and the fermions so as to spontaneously obtain the fermion mass $m$. Although the CJT potential $V(T, \mu, m)$ and the auxiliary scalar field effective potential $V_{\sigma}(T, \mu, m)$ may have different expressions, the key sector contained in the both are the same. That is the derived Schwinger-Dyson equation (31) from the thermal condensates $\langle\bar{\psi} \psi\rangle_{T}$.

\section{CONCLUSIONS}

We have proven that in a 4D NJL model, the CJT effective potential based on composite operators and the effective potential based on an auxiliary scalar field are completely equivalent not only at $T=\mu=0$ but also at a finite $T$ and $\mu$ if the momentum cutoff in the fermion loop integrals is large enough. The two effective potentials may give the same possible spontaneous symmetry (including chiral symmetry) breaking at $T=\mu=0$ and a low $T$ and $\mu$, and symmetry restoration at a finite $T$ and $\mu$. Although the expressions of the two effective potentials are different, the key sectors of the both are the same, it is the derived Schwinger-Dyson equation originated from the fermion -antifermion condensates. In particular, when the above equation is used, the two potentials can be transformed to each other. The discussions also imply that, in general, an effective potential, at least the one containing a single order parameter, is essentially determined by the Schwinger-Dyson equation corresponding to the extreme condition of the effective potential. This is because the mathematical form of the SD equation will determine the effective potential's extreme value points, maximums, minimums, etc. and they are essential for research on spontaneous symmetry breaking. Hence, corresponding to a given form of the SD equation, it seems that we are allowed to have different expressions for the corresponding effective potential [8]. 


\section{Acknowledgments}

This work was supported by the National Natural Science Foundation of China.

[1] W. Heisenberg and H. Euler, Z. Phys. 98, 714 (1936); J. Schwinger, Phys. Rev. 82, 664 (1951).

[2] J. Goldstone, A. Salam, and J. Weinberg, Phys. Rev. 127, 965 (1962); G. Jona-Lasinio, N. Cimento 34, 1790 (1964).

[3] S. Coleman and E. Weinberg, Phys. Rev. D7, 1888 (1973).

[4] R. Jackiw, Phys. Rev. D9, 1686 (1974).

[5] J. Iliopoulos, C. Itzykson, and A. Martin, Rev. Mod. Phys. 47, 165 (1975).

[6] Y. Nambu and G. Jona-Lasinio, Phys. Rev. 122, 345 (1961); 124, 246 (1961).

[7] D. Ebert and H. Reinhardt, Nucl. Phys. B271, 188 (1986); B. Rosenstein, B. J. Warr, and S. H. Park, Phys. Rep. 205, 59 (1991) and references therein; V. A. Miransky, Dynamical symmetry breaking in quantum field theory (World Scientific, Singapore, 1993).

[8] Zhou Bang-Rong, Commun. Theor. Phys. 39, 663 (2003).

[9] J. M. Cornwall, R. Jackiw, and E. Tomboulis, Phys. Rev. D10, 2428 (1974).

[10] K. Higashijima, Prog. Theor. Phys. Suppl. 104, 1 (1991).

[11] N. P. Landsman and Ch. G. van Weert, Phys. Rep. 145, 141 (1987).

[12] Bang-Rong Zhou, Phys. Rev. D57, 3171 (1998); Commun. Theor. Phys. 32, 425 (1999).

[13] Bang-Rong Zhou, Phys. Rev. D59, 065007(1999); D62, 105004 (2000); D65, 027701 (2002); Commun. Theor. Phys. 37, 685 (2002).

[14] O. Kiriyama, M. Maruyama, and F. Takagi, Phys. Rev. D62, 105008 (2000).

[15] Zhou Bang-Rong, Commun. Theor. Phys. 40, 67 (2003); 40, 669 (2003).

[16] H. Reinhardt and B. V. Dang, J. Phys. G13, 1179 (1987). 